\title{
Divergences et convergences entre Max Weber et Ernst Troeltsch dans l'approche du protestantisme
}

\author{
Peter-Ulrich Merz-Benz
}

\section{(2) OpenEdition \\ 1 Journals}

Édition électronique

URL : http://journals.openedition.org/assr/1051

DOI : 10.4000/assr.1051

ISSN : 1777-5825

\section{Éditeur}

Éditions de l'EHESS

\section{Édition imprimée}

Date de publication : 1 juillet 2004

Pagination : $57-77$

ISBN : 2-222-96751-1

ISSN : 0335-5985

\section{Référence électronique}

Peter-Ulrich Merz-Benz, « Divergences et convergences entre Max Weber et Ernst Troeltsch dans l'approche du protestantisme », Archives de sciences sociales des religions [En ligne], 127 | juillet septembre 2004, mis en ligne le 25 juin 2007, consulté le 01 mai 2019. URL : http:// journals.openedition.org/assr/1051; DOI : 10.4000/assr.1051 


\section{DIVERGENCES ET CONVERGENCES ENTRE MAX WEBER ET ERNST TROELTSCH DANS L'APPROCHE DU PROTESTANTISME*}

La thèse de Max Weber sur le protestantisme compte parmi les approches les plus importantes, dès lors qu'il s'agit d'expliquer la naissance du monde moderne ou, pour être plus précis, la constitution de "la puissance la plus décisive de notre vie moderne : celle d[u] capitalisme" (1). Pour Max Weber, il est établi que, venant compléter les "conditions économiques", le capitalisme en gésine "dépend également de la faculté et de la disposition des hommes à conduire de manière praticorationelle leur existence selon des voies spécifiques" (2) ; quant aux "éléments qui les forment", ces manières de conduire son existence renvoient à des "forces religieuses", en particulier au protestantisme (3). La manière de conduire son existence qui représente au plus près la singularité du capitalisme est le travail professionnel (Berufsarbeit). En pensée et en actes, une importance singulière revient au travail professionnel, lequel est, dans la conception protestante, au fondement de l'accès au salut. Par le passé, cette thèse de Weber a fait l'objet de nombreuses recherches en histoire et dans les sciences sociales; un point décisif du problème est néanmoins demeuré ouvert jusqu'à aujourd'hui.

Dans ses travaux de sociologie des religions, les prises de position de Weber à l'égard de l'œuvre du théologien, historien des religions, philosophe de l'histoire et sociologue, Ernst Troeltsch, sont connues et également documentées. Moins connu, pour ne rien dire de son examen systématique et de fond, en revanche est le fait que, dans ses écrits, Ernst Troeltsch a développé une thèse "propre" sur le protestantisme - sur le rapport entre le protestantisme et le monde moderne -, thèse qu'il est tout à fait permis d'entendre comme une alternative à la thèse correspondante de Weber. D'une manière qu'on peut expliquer, Troeltsch voit d'emblée le protestantisme comme faisant partie d'un ensemble de faits relevant de l'histoire

* Traduit de l'allemand par Alexandre Escudier

(1) WEBER, 1988a, p. 4.

(2) WeBER, 1988a, p. 16.

(3) WeBer, 1988a, p. 16. 
universelle, en quoi il attribue également au protestantisme en tant que tel une importance du point de vue de l'histoire universelle; à l'inverse, eu égard à l'influence qu'il a exercée sur la manière dont les hommes ont conduit leur existence, Weber veut avant tout voir dans le protestantisme un phénomène singulier, n'acquérant d'importance du point de vue de l'histoire universelle, qu'à la suite du capitalisme, lequel, dans l'ensemble, revêt les dimensions d'un destin pour le monde moderne. Ce qui rend particulièrement intéressant et riche d'informations le lien établi par Weber et Troeltsch entre le protestantisme et le monde moderne, est le fait que, dans leurs tentatives d'explication, ces deux auteurs utilisent le même fondement méthodique, à savoir: la logique de l'histoire de Heinrich Rickert. Ainsi, à partir d'une base commune, il est possible de dégager la structure logique ainsi que le contenu de chacune des thèses sur le protestantisme et de mettre en visà-vis chacune des deux variantes de la thèse en présence. Les développements qui vont suivre ont pour but de livrer quelques éléments d'information à ce sujet.

Je commencerai avec quelques remarques sur le contexte dans lequel ces deux thèses sur le protestantisme sont apparues (I). Ensuite, je me pencherai sur la thèse de Weber sur le protestantisme; en suivant les éléments avancés par Weber luimême, il s'agira de spécifier le rapport entretenu par le protestantisme et le capitalisme en tant que construction idéal-typique d'un processus évolutif réel (II). Dans une troisième partie sera exposée la thèse de Troeltsch sur le protestantisme: sa reconstruction, en termes d'histoire universelle, du complexe causal qui relie le protestantisme au monde moderne (III). Enfin, mon attention se portera sur les points communs et les différences constatables entre ces deux thèses (IV).

\section{I - Le contexte historique d'émergence des thèses wébérienne et troeltschienne sur le protestantisme}

En 1904 et 1905, paraît dans la revue Archiv für Sozialwissenschaft und Sozialpolitik la première version de l'étude de Max Weber intitulée "L'éthique protestante et 'l'esprit' du capitalisme" (4). Peu de temps après, Max Weber reçoit de la part de la Société des historiens d'Allemagne la demande d'exposer les résultats de son étude lors de la prochaine session annuelle de la Société, prévue pour avril 1906. Weber fait suivre cette demande à son collègue et ami d'Heidelberg, Ernst Troeltsch. Ce dernier profite alors de l'occasion, lors d'un exposé long et érudit, pour faire mieux comprendre au monde scientifique ses positions sur "L'importance du protestantisme dans l'émergence du monde moderne". Cet exposé est publié la même année dans l'Historische Zeitschrift (5); une seconde version augmentée paraît comme monographie en 1911 (6). La raison pour laquelle Weber n'a pas donné personnellement suite à la demande qu'on lui adressait

(4) Weber, 1904 ; Weber, 1905. Dans ce qui suit, cette étude sera citée d'après sa réédition de 1993 ; WEBER, 1993 [1904-1905].

(5) Troeltsch, 1906.

(6) Troeltsch, 1911. 
demeure incertaine. Dans une note de son exposé, Troeltsch fait sur ce point remarquer que Weber en a "malheureusement été empêché par d'autres travaux" (7). Mais on peut tout aussi bien émettre l'hypothèse que, suite à l'épreuve de la maladie qu'il avait dû traverser au cours des années précédentes, Weber ne se sentait pas encore en mesure d'affronter un public large et exigeant tel que celui rassemblé par un Congrès d'historiens (8). Plus importantes que les raisons pour lesquelles Weber a été empêché, sont les conséquences, résultant de la prise en charge par Troeltsch de l'exposé, quant à la manière de traiter le thème proposé. Car sans aucune ambiguïté et dans la mesure où il travaille lui aussi sur le thème 'protestantisme et monde moderne', Troeltsch prévoit en fait d'expliciter des vues qui lui sont propres, et, en conséquence, de se démarquer de Weber. Au regard des réponses que Weber formulera par la suite - souvent en langage codé -, les propositions avancées par Troeltsch dans ce cadre donnent déjà, par comparaison, une idée précise des différences de fond existant entre leurs deux thèses sur le protestantisme.

Dans la note déjà mentionnée de l'exposé qu'il fait en 1906 devant le Congrès des historiens allemands, Troeltsch concède - en se référant explicitement à Weber - qu'il n'est "pas en mesure de traiter en spécialiste des aspects politiques, économiques et sociaux du thème retenu" : il concède qu'il ne s'est, quant à lui, “occupé que des idées développées par le protestantisme ancien sur l'État, l'Église et la culture, qui plus est, qu'aux constellations philosophico-scientifiques et religieuses" (9). "Pour ce qui est de préciser et d'étayer plus avant" les résultats mis au jour par ses recherches, il renvoie à la "contribution" qu'il a donnée au recueil Culture du présent : c'est-à-dire à son texte intitulé "Christianisme protestant et Église des temps modernes" où sont consignés ses travaux antérieurs sur le thème "Protestantisme et monde moderne"(10). Troeltsch signale ainsi que lui-même s'était déjà penché sur le thème "Protestantisme et monde moderne", ce fait étant attesté dans certaines de ses publications, antérieures de plusieurs années au Congrès des historiens allemands au cours duquel il a été appelé à remplacer Weber. En plusieurs points de son exposé, Troeltsch exprime son accord avec son collègue d'Heidelberg et fait référence à la thèse de Weber sur le protestantisme ; il profite néanmoins de l'occasion pour émettre simplement une petite réserve. Cette réserve est néanmoins d'une importance extrême. Troeltsch y affirme sans aucune ambiguïté qu'il est une chose à laquelle Weber "est parfaitement parvenu" : il a "apporté la preuve" que “c'est l'ascèse spécifiquement calviniste qui (...) a permis l'essor d'une complexion spirituelle sur le fond de laquelle le capitalisme a pu se déployer de façon si brutale et en fait si contraire à l'ordre naturel" (11). Et Troeltsch de continuer : pourtant, "peut-être est-il permis (...) de davantage souligner le fait (...) que ce type particulier de l'ascèse réformée du travail [note de l'Auteur : l'ascèse calviniste] a aussi été codéterminé par les conditions propres à

(7) Troeltsch, 1906, p. 1 (note 1).

(8) Lehmann, 1996a, p. 10.

(9) Troeltsch, 1906, p. 1 (note 1).

(10) Troeltsch, 1906, p. $1 \mathrm{sq}$. (note 1). «Die Kultur der Gegenwart, Teil I Abteilung IV.I. Hälfte » ainsi qu'une partie de ce texte, sous le titre « Protestantisches Christentum und Kirche der Neuzeit », ont paru le 20 janvier 1906 ; Ernst Troeltsch, Bibliographie, 1982, p. 74.

(11) Troeltsch, 1906, pp. 45 et $43 \mathrm{sq}$. 
l'état des affaires en Occident, notamment l'effacement du différend existant entre l'État et la culture étatique" (12). Si cela est exact et si, vu son importance dans l'émergence du monde moderne, l'ascèse réformée du travail a bien été codéterminée par des forces économiques et politiques - ce sur quoi Troeltsch tient expressément à "mettre l'accent" -, ladite ascèse s'inscrirait alors directement dans le faisceau général des relations causales individuelles ayant eu part à l'émergence $\mathrm{du}$ monde moderne. D'une importance majeure du protestantisme, du protestantisme en tant qu'il constitue le socle sur lequel s'est formée "la puissance la plus décisive de la vie moderne" (13) - ainsi que l'avance Weber -, il ne peut dès lors plus guère être question. Ce résultat étant de surcroît confirmé par le fait que la relation mise au jour par Weber, après Troeltsch, entre le protestantisme, ou plutôt le calvinisme, et le capitalisme moderne se trouve circonscrite au domaine de l'économie - alors que, pour Troeltsch, le domaine de l'économie jouxte à un degré d'importance égal les domaines de la "famille", du "droit", de "l'État", de la "société", de la "science" et de "l'art" (14). Selon Troeltsch, le rapport du calvinisme au capitalisme moderne tel que Weber le conçoit, ne constitue donc plus une relation causale historique singulière parmi d'autres; il apparaît simplement comme un moment de l'évolution historique d'ensemble conduisant à l'émergence du monde moderne. L'importance du protestantisme dans l'émergence du monde moderne du point de vue de l'histoire universelle, c'est bien là la problématique d'Ernst Troeltsch; et en cela la réserve formulée par Troeltsch à l'égard de Weber ne signifie au fond rien moins que le fait qu'il insère pour ainsi dire la thèse wébérienne sur le protestantisme à l'intérieur de sa propre thèse.

Max Weber ne s'est pas exprimé sur la réception par Troeltsch de sa thèse sur le protestantisme - à tout le moins jamais directement. Dans le cadre de la controverse autour de Rachfahl, certaines de ses assertions semblent néanmoins tout à fait remarquables. En 1909, l'historien de Kiel, Felix Rachfahl, publie dans l'Internationale Wochenschrift une étude volumineuse intitulée "Calvinisme et capitalisme ", dans laquelle Weber et Troeltsch, mais surtout Weber, sont pris à partie de la manière la plus violente (15). Là-dessus, Weber rédige immédiatement une "anti-critique", dans laquelle il se défend tout d'abord avec véhémence d'être considéré avec Troeltsch comme faisant partie d'un même "collectif" (16). Weber tire le point au clair en ces termes : lorsque Troeltsch "s'est référé à certains de [ses] développements, il s'est toujours agi (...) de zones de contact, périphériques quant au problème qui le retient, de ses résultats de recherche avec les miens" (17). Au reste, ses "travaux sur ces choses", c'est-à-dire sur le calvinisme et le capitalisme, il les a - ajoute Weber - "déjà pour partie exposés, il y a aujourd'hui douze ans [en 1897], dans notre séminaire" (18). Pour ce qui est de ce "séminaire", il s'agit à l'évidence d'un des premiers enseignements donnés par Weber à Heidelberg; et les "travaux" dont Weber parle ici concernent vraisemblablement sa

(12) Troeltsch, 1906, p. 45.

(13) WEBER, 1988a, p. 4.

(14) Troeltsch, 1906, pp. 29-56.

(15) RachFAhL, 1909, cité d'après la réimpression de 1987.

(16) WeBER, 1910, cité d'après la réimpression de 1987, p. 149.

(17) WEBER, 1987 [1910], p. 150.

(18) WeBER, 1987 [1910], p. 150. 
réception du livre de Eberhard Gothein intitulé Histoire économique de la Forêt Noire, paru en 1892. C'est Gothein qui, dans son ouvrage, a formulé une conclusion des plus intéressantes pour Weber, à savoir que "la diaspora calviniste [est] en même temps la pépinière de l'économie capitaliste" (19). En se référant encore une fois à Troeltsch, Weber affirme que ce dernier "déploie le processus historique de constitution des doctrines sociales des Églises chrétiennes" - alors que lui “n'a jusqu'à présent cherché qu'à rendre sensible, et selon sa contingence religieuse (originelle), un phénomène particulier quant à la manière qu'ont les hommes de conduire leur existence [note de l'Auteur : à savoir, le travail professionnel de type capitaliste]" (20). C'est là à vrai dire une façon extrêmement 'sélective' de caractériser le travail de Troeltsch. Car ce faisant, Weber mentionne seulement l'ouvrage de Troeltsch, paru entre 1908 et 1910, sous la forme d'une série d'articles, sur Les doctrines sociales des Églises et des groupements chrétiens (21), et passe totalement sous silence le fait que Troeltsch a développé une thèse propre sur le protestantisme. Cela est d'autant plus étonnant que Rachfahl avait expressément dirigé sa critique contre les travaux de Weber et de Troeltsch consacrés au thème protestantisme et capitalisme, ou plutôt protestantisme et monde moderne (22). Or, chez Rachfahl, il n'est à nul endroit question des Doctrines sociales de Troeltsch.

Peu de temps après Max Weber, c'est au tour de Troeltsch de se positionner par rapport aux reproches avancés par Felix Rachfahl. Et de son côté, Troeltsch souligne, lui aussi, qu'il ne constitue pas avec Weber "une seule et même firme scientifique" (23). C'est d'une manière tout à fait remarquable que Troeltsch se distingue derechef de Weber. Les “travaux de Max Weber sur '1'esprit du capitalisme et l'éthique protestante' doivent [selon Troeltsch] être compris" (24) selon la perspective du "problème qu'il y a à élucider le rapport qu'entretient 'l'infrastructure' socio-économique réelle de l'évolution propre aux temps modernes avec sa 'superstructure' idéologique scientifico-éthico-religieuse" (25). Quant à ses propres recherches, il s'agirait "essentiellement d'histoire des religions", par quoi, "naturellement", il "[aurait] été amené à objectiver les relations de dépendance entretenues par l'élément religieux avec les conditions réelles d'existence ainsi qu'inversement l'action exercée par celles-ci sur celui-là" (26). “À l'encontre de l'importance trop grande ordinairement accordée à l'influence culturelle exercée par le protestantime, [il] aurait là tenté d'apporter la preuve que [note de l'A. : cette influence] est relative et limitée, tout en faisant par ailleurs ressortir l'effet positif qu'a été la sienne" (27). Dans la thèse de Weber sur le protestantisme, Troeltsch voit le "cas particulier" d'une évolution au cours de laquelle des phénomènes économiques et sociaux "se trouvent" tout d'abord "influencés et imprégnés par une sphère idéelle avant de reprendre l'ascendant et de rétroagir sur cet élément idéologique" (28).

(19) Gothein, 1892, p. 674.

(20) WeBer, 1987 [1910], p. 150.

(21) Pour les références détaillées, cf. Ernst Troeltsch, Bibliographie, 1982, p 89.

(22) RACHFAHL, 1987 [1909], p. 58.

(23) Troeltsch, 1910, cité d'après la réimpression de 1987, p. 189.

(24) Troeltsch, 1987 [1910], p. 189.

(25) Troeltsch, 1987 [1910], p. 189.

(26) Troeltsch, 1987 [1910], p. 191.

(27) Troeltsch, 1987 [1910], p. 191.

(28) Troeltsch, 1987 [1910], p. 190. 
Les résultats de cette "analyse historico-génétique" de Weber (29), Troeltsch les a "pour l'essentiel" repris - ainsi qu'il le constate de manière explicite (30). D'une manière à vrai dire extrêmement révélatrice, Troeltsch ajoute alors ceci : "Ses conclusions [note de l'A. : celles de Weber], je les ai simplement inscrites dans un autre cadre, défini à partir de ma visée propre de connaissance" (31).

Mais la thèse de Max Weber sur le protestantisme a-t-elle effectivement trait à un problème relevant "purement de l'histoire économique" (32), ainsi que Troeltsch l'avance? Weber comprend-il effectivement le rapport du calvinisme au capitalisme - toujours selon la caractérisation de Troeltsch - comme un "phénomène" historique "singulier" ? (33) Enfin et au bout du compte, la thèse de Weber sur le protestantisme se laisse-t-elle "intégrer" (34) au complexe causal, étudié par Troeltsch, qui relie le protestantisme au monde moderne - étant entendu que ce complexe causal ferait partie d'un "ensemble" (35), à savoir de ce cadre général où "l'élément religieux du protestantisme est envisagé selon la position qu'il occupe par rapport aux systèmes historico-culturels environnants"? (36) Afin d'élucider ces questions, il nous faut maintenant nous pencher directement sur les deux thèses en présence et que nous nous appliquions à exposer la structure logique ainsi que le contenu de la thèse de Max Weber sur le protestantisme.

\section{II - Structure logique et contenu des thèses de Max Weber sur le protestantisme}

Quelle est l'objectif poursuivi par Max Weber dans son texte intitulé L'éthique protestante et l'esprit du capitalisme? Dans cette étude, Weber ne cherche en aucune manière à donner une réponse globale à la question de l'origine et de la spécificité historiques du capitalisme moderne ni à celle de savoir s'il a forcé de destin, ainsi qu'on le présume souvent. Weber ne considère pas davantage que sa thèse sur le protestantisme soit une réponse aux interrogations de Marx; Weber s'est expressément défendu de vouloir remplacer le caractère unilatéral d'une "vision" purement "économique de l'histoire" par une conception "idéaliste", voire "spiritualiste", de l'histoire. Il rejette également avec force la "thèse folle et doctrinaire" selon laquelle "l'esprit capitaliste (...) [aurait] seulement pu naître comme émanation de certaines influences de la Réforme" (37); lui demeure également

(29) Troeltsch, 1987 [1910], p. 191.

(30) Troeltsch, 1987 [1910], p. 191.

(31) Troeltsch, 1987 [1910], p. $191 s q$.

(32) Troeltsch, 1987 [1910], p. 190 ; cf. ibid., p. 192.

(33) Troeltsch, 1987 [1910], p. 192.

(34) Troeltsch, 1987 [1910], p. 192.

(35) Troeltsch, 1987 [1910], p. 192.

(36) Troeltsch, 1987 [1910], p. 192.

(37) Weber, 1993 [1904-1905], pp. 50 sq. Une partie des affirmations de Weber a été laissée de côté : elle constitue l'objet même d'un ajout opéré plus tard par Weber, que je cite moi-même ci-après. 
étrangère l'idée "qu'en tant que système économique le capitalisme serait un produit de la Réforme" (38). Il s'agit seulement - ainsi que Weber l'écrit luimême - "d'établir si et dans quelle mesure (...) des influences religieuses ont effectivement pris part à l'élaboration qualitative et à l'expansion quantitative [de 'l'esprit' du capitalisme (note de l'A.)] ainsi que de constater quels aspects concrets de la culture capitaliste sont référables auxdites influences" (39). La seule chose qui importe ici à Weber ce sont les "affinités électives entre certaines formes de la foi religieuse" et l'esprit capitaliste, entre le protestantisme et "l'éthique professionnelle" (Berufsethik) propre au capitalisme (40). Weber cherche à présenter cette action exercée par des "mobiles religieux" sur l'éthique professionnelle propre au capitalisme, sous la forme d'un idéaltype, plus précisément sous la forme de la construction idéaltypique d'une évolution historique.

Mais pourquoi Weber recourt-il à la notion d'idéaltype ? Et que doit-on entendre sous le terme d'idéaltype ? Une évolution historique réelle renferme une série d'individus historiques qui se succèdent les uns aux autres, une série d'états singuliers de ce qui advient dans le temps; ces individus historiques sont reliés entre eux, pour ainsi dire, par des rapports d'influence causaux individuels, ils sont les produits de ces rapports d'influence. Il s'agit là du concept classique de développement historique tel qu'il a été forgé par Heinrich Rickert dans son livre intitulé Les limites de la conceptualisation dans les sciences de la nature ["Die Grenzen der naturwissenschaftlichen Begriffsbildung"] (41) et que Weber a repris sans la moindre réserve (42). Accéder à la connaissance d'évolutions historiques revient ainsi à être en mesure d" assigner" des causes concrètes à des effets concrets ; à partir de pans déterminés de la réalité présente, il convient - par régression causale - de trouver une voie qui permette d'établir les causes responsables de l'évolution desdits pans de réalité. Pour parvenir à assigner de la sorte des causes concrètes à des effets concrets, on a besoin de l'idéaltype (43). L'idéaltype renferme l" "image idéale" de ce qui a conduit à une évolution historique ; l'idéaltype présente en cela l' "image idéale d'une transformation", d'une transformation qui, elle, ne s'est incarnée que de manière allusive et partielle dans les événements singuliers qui constituent le déroulement réel des faits, déterminé de manière causale. Ce qui n'apparaît que de manière indistincte dans une évolution historique réelle reçoit, dans l'idéaltype, ses contours purs et entiers. L'idéaltype est un "tableau de pensées", voire un "tableau imaginaire" (44), de ce qui dans la réalité ne s'incarne que de manière extrêmement imparfaite et allusive. C'est cette dimension fictionnelle de l'idéaltype qui est en fait décisive. Lorsqu'une évolution historique réelle se trouve pour ainsi dire 'confrontée' à son idéaltype, des correspondances éventuelles apparaissent; Weber écrit lui-même ceci : "Afin de comprendre les constellations causales réelles, nous en construisons

(38) WeBEr, 1988b, p. 83. Cet ajout provient de la seconde version de l'étude sur le protestantisme, parue en 1920 dans le cadre des Gesammelte Aufsätze zur Religionssoziologie, vol. 1.

(39) WEBER, 1993 [1904-1905], p. 51.

(40) WEBER, 1993 [1904-1905], p. 51.

(41) Rickert, 1902, p. 437 ainsi que pp. 579 sq. ; Merz-Benz, 1990, § 8, en particulier p. 193 sq., $\S 10$, en particulier p. $211 s q$.

(42) Cf. sur ce point, de manière générale, Merz-Benz, 1990, pp. 371 sq. ainsi que le § 13c.

(43) Weber, 1973, p. 203 ; cf. MerZ-BenZ, 1990, p. 403 sq.

(44) WEBER, 1973, p. 191 sq., $275 ; 277$. 
d'irréelles" (45); ce sont de telles constellations causales irréelles que renferme l'idéaltype. La thèse de Weber sur le protestantisme n'est pas une explication causale; elle est une construction logico-méthodique dont la vocation est de susciter des explications causales.

En tant que construction logico-méthodique, l'idéaltype est un apport propre de Weber, une notion à vrai dire composée d'éléments empruntés à la logique rickertienne de l'histoire ; seul le terme d" "idéaltype" provient, comme on sait, de Georg Jellinek. Parmi les éléments constitutifs des idéauxtypes, j'aimerais ici nommer les plus importants (46), à savoir : 1) les conceptions axiologiques qui président à la constitution de l'ensemble des faits historiques envisagés; selon leur forme pure et entière, ces conceptions axiologiques sont pensées comme déterminant les mobiles et les calculs des acteurs; 2) un idéaltype est le produit de l'intégration, de 1'“agglomération idéelle en soi homogène" (47) des éléments d'action susceptibles de révéler la parfaite réalisation des contenus axiologiques concernés. Pourquoi telles actions déterminées résultent-elles de telles conceptions axiologiques déterminées, entendues comme autant de mobiles à agir - c'est ce qui est exposé dans l'idéaltype, d'une manière pure, compréhensible, et, pour Weber, sous une forme rationnelle.

Demandons-nous maintenant en quoi consiste la construction idéaltypique de la thèse sur le protestantisme ? Le mobile qui détermine les calculs des acteurs est "l'esprit capitaliste"; la conception axiologique qui satisfait à ce mobile est l'éthique professionnelle calviniste ; et le complexe d'actions qui révèle la réalisation parfaite de cette conception axiologique, voire de ce mobile, ce sont les fondements de l'approche puritaine, née du calvinisme, de la vie professionnelle et de la vie active. Demeure également l'apport méthodique que représente cette thèse sur le protestantisme: si l'on confronte l'évolution réelle, en termes d'histoire économique, avec cette thèse sur le protestantisme, il est désormais possible, d'une part, de déterminer dans quelle mesure les principes de l'agir économique de type puritain représentent un dépassement de l'économie traditionnelle axée sur la satisfaction des besoins; et sur cette base - en prenant pour fil directeur le rapport de l'agir économique puritain à l'esprit capitaliste -, deviennent, d'autre part, perceptibles de possibles stimulants de l'évolution économique qui ne sont explicables ni par les causes économiques ni par la pensée économique traditionnelle, purement orientée vers la notion de besoin.

Mais comment les éléments de la thèse sur le protestantisme sont-ils constitués dans le détail ? Selon Weber, on entend "parle[r] l'esprit du capitalisme de manière caractéristique" dans les Necessary hints to those that would be rich (48) formulés par Benjamin Franklin en 1736. Ces "utiles indications" - ainsi que Weber l'ajoute aussitôt - sont à vrai dire une chose bien singulière. Car ce qui se trouve "enseigné" à travers elles, ce n'est en aucune manière le simple "sens des affaires", une simple 'technique' du succès économique; en elles c'est un "éthos"

(45) WeBER, 1973, p. 287.

(46) Pour un exposé exhaustif de la logique présidant à la construction des idéauxtypes, cf. MerzBENZ, 1990, §§ 15ba-15bc.

(47) WeBER, 1973, p. 191.

(48) WeBER, 1993 [1904-1905], pp. 13 et 12 sq. 
propre (49) qui “s'exprime". Et le "summum bonum" de cette éthique, c'est - écrit Weber - "gagner de l'argent, toujours plus d'argent, en évitant strictement toute jouissance spontanée, (...) entièrement dépris de tout point de vue eudémoniste ou même hédoniste, (...) [le fait de gagner de l'argent] étant purement pensé comme une fin en soi" (50). Quiconque connaît le succès comme capitaliste moderne (51) ne s'adonne par conséquent pas à cette tâche pour soi-même, mais remplit un "devoir" qui lui est imposé (52). En ce sens, "un certain caractère ascétique" (53) convient à la "manière de conduire son existence" propre à "l'entrepreneur capitaliste", en tant qu'il est le véhicule idéaltypique de l'esprit capitaliste : l'entrepreneur capitaliste “"ne tire rien' de sa richesse pour lui-même - en dehors du sentiment irrationnel d'avoir bien 'accompli sa besogne'" (54). Imposée par le "devoir" de s'adonner avec succès à une activité professionnelle et économique, cette tâche renvoie à l'éthique protestante, à l'éthique professionnelle calviniste ; l'éthique professionnelle calviniste est une conception axiologique qui satisfait au mobile du travail professionnel capitaliste.

Dès le début de la seconde partie de son étude sur L'éthique protestante et l'esprit du capitalisme, Weber marque clairement ce qui lui importe dans son travail sur le protestantisme, et principalement sur le calvinisme : "il s'agit de mettre au jour ces motivations psychologiques, nées de la croyance religieuse et de la pratique de la vie religieuse, et qui [impriment] aux conduites humaines leur direction et y [maintiennent] l'individu" (55). En cela, il s'agit principalement de se concentrer sur la "doctrine" calviniste "de la prédestination" (Gnadenwahl) (56). Conformément à une décision absolument libre de Dieu, il est établi "de toute éternité" quelle "partie de l'humanité sera sauvée" et quelle autre "demeurera damnée" (57). Les hommes n'ont, quant à eux, connaissance que de l'existence de ce partage ; Dieu seul sait par contre qui fait ou non partie des élus (58). Et comme c'est là "une chose impensable" que de "considérer" les décrets de Dieu "comme modifiables par l'intervention humaine", ni le "mérite ni la culpabilité" des hommes ne sauraient avoir une influence quelconque sur [la] "fatalité" de l'élection (59). Mais dans sa solitude intérieure et son état d'incertitude - et c'est là pour Weber le "problème décisif" - comment l'individu isolé peut-il pourtant "supporter" cette doctrine ? (60) Existe-t-il un moyen de faire front aux "tourments suscités" par la doctrine calviniste de la prédestination (61), flanquée de son "inhumanité pathétique" ? (62) Répondre à ces questions nous conduit rien moins qu'à l'idée

(49) Weber, 1988b [1920], p. 33 ; cf. Weber, 1993 [1904-1905], pp. 13 et 15.

(50) Weber, 1993 [1904-1905], p. 15 ; Weber, 1988b [1920], p. 35.

(51) WEBER, 1988b [1920], p. 34.

(52) Weber 1993, [1904-1905], p. 16 ; WeBER, 1988b [1920], p. 36.

(53) WEBER 1993, [1904-1905], p. 16 ; n’ont pas été ici signalés dans le texte les passages soulignés par Weber ; Peter-Ulrich Merz-BenZ; Weber, 1988b [1920], p. 36.

(54) Weber, 1993 [1904/5], p. 29 ; WeBER, 1988b [1920], p. 55.

(55) WeBer, 1988b [1920], p. 86 ; cf. WeBer, 1993 [1904-1905], p. 55.

(56) WeBER, 1993 [1904-1905], p. 57 sq. ; WEBER, 1988b [1920], p. 89 sq.

(57) Weber, 1993 [1904-1905], p. 61 ; Weber, 1988b [1920], p. 93.

(58) Weber, 1993 [1904-1905], p. 61 ; Weber, 1988b [1920], p. 93.

(59) Weber 1993 [1904/-1905], p. 61 ; Weber, 1988b [1920], p. 93.

(60) WeBER, 1993 [1904-1905], p. 68 ; WEBER, 1988b [1920], p. 102.

(61) WeBER, 1993 [1904-1905], p. 70 ; WEBER, 1988b [1920], p. 105.

(62) Weber, 1993 [1904-1905], p. 62 ; Weber, 1988b [1920], p. 93. 
fondamentale de la thèse wébérienne sur le protestantisme : la doctrine inflexible de la prédestination demeure certes inentamée, mais le croyant dispose des moyens de se procurer "la certitude subjective de sa propre élection" (63). Et le "meilleur moyen" "d'accéder à cette confiance en soi" est de s'adonner "sans relâche à une activité professionnelle" (64). Une chose doit être clairement soulignée : avec toutes les performances qu'elle requiert d'accomplir, l'activité professionnelle ne constitue pas le moyen d'acheter son salut (65); quant à l'élection d'un individu, cela demeure pour ce dernier une décision insondable qui relève de Dieu. Mais, pour le croyant, l'activité professionnelle est le moyen, le "moyen technique (...) de se délivrer de l'angoisse du salut" (66), le "moyen technique" de constamment combattre cette angoisse en lui-même. Pourquoi Weber parle-t-il ici lui-même de "technique" ? Quiconque, pour obtenir son salut, ne peut être crédité de "bonnes œuvres" (67) et demeure par conséquent toujours dans l'incertitude quant à son état d'élection: il se trouve confronté “à chaque instant à l'alternative suivante : être élu ou réprouvé ? (68)" Dans cette situation, s'adonner sans relâche à une activité professionnelle aide le croyant, non pas sous la forme d'un affairement aveugle et irréfléchi, mais seulement en tant que ceci constitue une "méthode" conséquente, systématiquement élaborée, propre à "entièrement" déterminer la "conduite d'une existence" (69). Celui qui sait se contrôler à tout moment de son existence pourra surmonter son angoisse du salut de manière optimale (70). En retour, seule cette attitude apporte la garantie d'une conduite de part en part systématisée, et en ce sens "rationalisée", quant à son déroulement intramondain (71). En tant que "méthode déterminant entièrement la conduite d'une existence", l'activité professionnelle est seule à même de "soulager de cette tension énorme dans laquelle vivre", "le destin inévitable des calvinistes, un destin qu'il n'est aucun moyen d'adoucir" (72). Et en cela l'activité professionnelle ne cesse d'apparaître comme une 'piété vécue', s'accomplissant ici-bas quoiqu'exclusivement "dirigée vers une fin transcendante : le salut" (73). Le croyant ne tient en rien à la jouissance de la vie, à la consommation de ce qu'il a lui-même produit ; tout ce qui lui importe, c'est de constamment toucher à la certitude du salut. Dans cette mesure, un "caractère spécifiquement ascétique" (74) convient à la "manière qu'il a de conduire son existence", et - d'après l'expression fameuse de Weber - sa vie quotidienne consiste en une "ascèse intramondaine" authentique (75). Via son activité professionnelle, via son agir économique, le croyant ne cherche finalement rien moins qu'à combler peu à peu le caractère insondable des modalités d'élection devant Dieu; c'est

(63) Weber, 1993 [1904-1905], p. 71 ; WeBER, 1988b [1920], p. 105.

(64) WEBER, 1993 [1904-1905], p. 71 ; WEBER, 1988b [1920], p. 105. pp. $74 s q$.

(65) WeBER, 1988b [1920], p. 110 ; ainsi que de façon complémentaire, WeBER, 1993 [1904-1905],

(66) WeBER, 1988b [1920], p. 110.

(67) Weber, 1988b [1920], pp. 110 et 114 ; WeBer 1993 [1904-1905], pp. 75 et 77.

(68) WEBER, 1993 [1904-1905], p. 75 ; WEBER, 1988b [1920], p. 111.

(69) WeBER, 1993 [1904-1905], p. 77 ; WeBER, 1988b [1920], p. 115.

(70) WEBER, 1993 [1904-1905], p. 75 ; WEBER, 1988b [1920], p. 111.

(71) WeBER, 1993 [1904-1905], p. 77 sq. ; WEBER, 1988b [1920], p. 115.

(72) WEBER, 1988b [1920], p. 114.

(73) WEBER, 1993 [1904-1905], p. 77 ; WEBER, 1988b [1920], p. 115.

(74) WebER, 1993 [1904-1905], p. 78; WeBER, 1988b [1920], p. 116.

(75) WEBER, 1993 [1904-1905], p. 80 sq. ; WEBER, 1988b [1920], pp. 118 sq. 
précisément cela qui confère à l'activité professionnelle son caractère de stimulant psychologique devant lequel chaque mobile de satisfaction terrestre des besoins perd par conséquent en couleur, à proportion de ce que l'ici-bas représente au regard de l'au-delà.

Pour ce qui est finalement de la réalisation parfaite de l'ascèse protestante intramondaine, elle aboutit - ainsi que Weber l'expose à partir de l'approche puritaine, née du calvinisme, de la vie professionnelle et de la vie active - à un "résultat extérieur", à savoir : à "la formation du capital par l'épargne ascétique forcée" (76). Supplantant définitivement l'éthique économique traditionaliste, la nouvelle manière d'acquérir des biens ne se trouve pas seulement libérée de ses relents de simple aspiration au gain ni rendue légale comme telle, elle se donne à voir désormais expressément comme quelque chose qui émane de la volonté de Dieu (77). Dans la mesure où omettre cette nouvelle manière d'acquérir des biens constitue "le premier et, par principe, le plus grave des péchés", à savoir "gaspiller son temps" (78), elle apparaît même comme une prescription divine. Eu égard à cette finalité 'supérieure' qu'est la certitude du salut, se trouve en revanche repoussé tout "emploi irrationnel de la propriété" (79). De la propriété, on ne doit pas "jouir spontanément" - pour ne rien dire de la "consommation de luxe" (80); son usage relève plutôt, et expressément, d'un "emploi utilitaire et rationnel voulu par Dieu, au profit de choses nécessaires, revêtant quelque utilité pratique" (81). Même la "production" de biens relevant de l'économie privée doit obéir à des objectifs 'supérieurs' - à mille lieues de toute aspiration à la richesse comme telle ou même à mille lieues de la pure et simple "cupidité instinctive" (82). "Déchaînement de l'aspiration au profit" et "refrènement" de la jouissance, formation du capital et épargne ascétique forcée, ces deux phénomènes vont de concert, se complétant l'un l'autre, jusqu'à aboutir à l'approche puritaine de la vie ; c'est cette approche puritaine de la vie qui "a veillé sur le berceau de 'l'homo œconomicus' moderne" (83).

Arrivé à ce stade, nous avons précisé les éléments constitutifs de la thèse wébérienne sur le protestantisme, aussi pouvons-nous passer à l'examen de la thèse d'Ernst Troeltsch sur le protestantisme.

(76) WeBER, 1993 [1904-1905], p. 147 ; WeBER, 1988b [1920], p. 192.

(77) Cf. WeBer 1993 [1904-1905], p. 145 ; WeBer, 1988b [1920], p. 190.

(78) WeBER, 1993 [1904-1905], p. 124 ; WEBER, 1988b [1920], p. 167.

(79) WEBER, 1988b [1920], p. 190 ; cf. WEBER, 1988b [1920], pp. $166 \mathrm{sq}$.

(80) WEBER, 1993 [1904-1905], p. 145 ; WEBER, 1988b [1920], p. 190.

(81) WeBER, 1993 [1904-1905], p. 145 sq. ; WeBER, 1988b [1920], p. 191.

(82) WeBer, 1993 [1904-1905], p. 146 ; WeBER, 1988b [1920], p. 191.

(83) WeBER, 1993 [1904-1905], p. 149 ; WEBER, 1988b [1920], p. 195. 


\section{III - Ernst Troeltsch, ses thèses sur le protestantisme et sa tentative de dépassement des problèmes induits par l'historisme}

Tout comme Max Weber, Ernst Troeltsch ne laisse subsister aucune ambiguïté quant à ses visées scientifiques. Dans son "enquête" sur «L'importance du protestantisme dans l'émergence du monde moderne ", la seule chose qui le préoccupe, est "d'exposer le complexe causal reliant le protestantisme au monde moderne" (84). La thèse de Troeltsch sur le protestantisme traite de l'explication causale individuelle de l'émergence d'un individu historique, à partir d'un état antérieur, d'un état de faits singulier. La problématique de Troeltsch consiste à faire de "l'importance effective du protestantisme dans l'émergence de la culture moderne" (85) un objet de la recherche historique. Aussi, Troeltsch rejette-t-il avec détermination l'idée de se voir imputer la volonté d'accorder au protestantisme une "importance normative" pour "la vie d'aujourd'hui" ou même la volonté de fonder un "jugement de valeur" sur la culture moderne ou le protestantisme (86). À vrai dire, et à la différence de Max Weber, Troeltsch est, par principe, également hostile à l'usage de "notions idéales" aux fins d'exposer des ensembles de faits historiques (87). Cela n'est pourtant pas une critique de Weber, puisque Troeltsch confère d'emblée aux notions idéales une fonction normative, ignorant purement et simplement le fait que des notions idéales peuvent avoir une fonction strictement logique. Pour Troeltsch, il n'est d'alternative qu'entre la 'recherche factuelle empirique' et le fait de 'fonder normativement des faits au moyen de notions idéales'. Dès son article de 1903 intitulé "Qu'est-ce que 'l'essence du christianisme'? ", Troeltsch s'était engagé avec détermination, contre Adolf von Harnack, pour que "l'on séparât plus strictement l'élément proprement historique et l'élément historicophilosophico-normatif" ayant trait à des "notions essentielles" ou précisément "idéales" (88). C'est pourquoi, dans sa thèse sur le protestantisme, il aspire exclusivement à l'élaboration de “concepts généraux, fondés [empiriquement] et historiquement, permettant de faire apparaître des situations effectives comme un tout" (89). Ces “concepts généraux, fondés empiriquement et historiquement", sont, pour être plus précis, des 'concepts de portée universelle subsumant des individus historiques', des concepts que Heinrich Rickert avait élaborés dans sa logique de l'histoire. Troeltsch constate lui-même sans aucune ambiguïté que "tout esprit familier de ces questions se rend compte de ce que l'épistémologie de Rickert se

(84) Troeltsch, 1911, p. 101 ; c'est moi qui souligne ; Peter-Ulrich Merz-Benz; Troeltsch, 1906, p. 65 .

(85) Troeltsch, 1911, p. 102 ; Troeltsch, 1906, p. 65.

(86) Troeltsch, 1911, p. 102 ; Troeltsch, 1906, p. 65.

(87) Troeltsch, 1911, pp. 24 sq. ; Troeltsch, 1906, p. 14.

(88) Troeltsch, 1913 [1903], en particulier p. 449 ; Troeltsch ,1911, pp. 24 sq. ; Troeltsch, 1906, p. 14.

(89) Troeltsch, 1911, p. 24 ; Troeltsch, 1906, p. 14. Dans la première version de la thèse de Troeltsch sur le protestantisme, il est question de "concepts historiques généraux", mais ce qui se trouve par là visé renvoie à la même chose que dans la seconde version. 
trouve au fondement de [sa] conceptualisation" (90). Troeltsch partage également avec Rickert les mêmes problèmes méthodologiques : comment peut-on former de tels concepts? Comment est-il même possible de mettre au jour un complexe causal reliant de tels individus historiques sans que le regard de l'observateur ne se perde dans la diversité des faits réels à rapporter auxdits concepts ? Dans la totalité du réel, il convient, selon Rickert, de lire et de dégager les faits pertinents eu égard aux différents concepts envisagés, donc d'opérer une sélection (91). C'est cette même voie qu'emprunte Troeltsch. Mais alors, quel est chez ce dernier le critère qui permet d'opérer une telle sélection? De fait, si Troeltsch recourt à un tel critère, il ne le fait pas de manière explicite. Il ne se trouve qu'une seule indication - qui, à vrai dire, en dit long : dans la seconde édition augmentée de sa thèse sur le protestantisme de 1911, Troeltsch affirme qu'il s'agit pour lui “d'exposer le complexe causal reliant le protestantisme au monde moderne (...), pour autant qu'il en existe un" (92). Ce qui signifie que Troeltsch ne cherche pas à exposer dans toute leur étendue ces phénomènes historiques que sont le protestantisme et le monde moderne. Il s'agit plutôt de saisir, à l'aide de concepts, ceux de leurs éléments constitutifs renvoyant à un complexe causal susceptible d'établir un lien entre lesdits phénomènes. C'est déjà à partir de ce point de vue que Troeltsch a envisagé le concept de monde moderne ; toute son attention se porte sur le trait réel distinctif de l'individualisme, c'est à partir de lui que s'ouvre la voie qui nous ramène au protestantisme. Mais, plus précisément, à quoi ressemble le concept de monde moderne tel que l'emploie Troeltsch?

Selon Troeltsch, pour définir le concept de monde moderne, "nous n'avons guère en tête que l'idée d'un contraste par rapport aux époques antérieures" (93) ; le concept de monde moderne se trouve donc "essentiellement" déterminé par défaut, ex negativo (94). Les caractéristiques constitutives du monde moderne "apparaissent" uniquement par opposition à la culture religieuse homogène du Moyen Âge, une culture forgée par le catholicisme (95). Le monde moderne : c'està-dire "en tous lieux le fait de combattre et [ensuite] de remplacer" la culture ecclésiale homogène par une multitude "d'idéaux culturels forgés de manière autonome" (96); ces idéaux culturels ne sont plus dominés par des "normes divines", mais sont en mesure de fonder eux-mêmes leur valeur, "par leur force de persuasion" (97). Que des idéaux culturels puissent eux-mêmes se donner un fondement, cela signifie ici essentiellement qu'il y va de la fondation de "convictions rationnelles" (98); en outre, c'est à l'action de la rationalité qu'il convient d'imputer la révélation du caractère historiquement contingent, donc relatif, des constructions culturelles forgées de manière autonome. À l'évidence, Troeltsch se

(90) Troeltsch, 1911, p. 25, note 1; Troeltsch, 1906, p. 14, note 1; Troeltsch, 1913 [1903], pp. 449 sq., note 32.

(91) Rickert, 1902, p. 306 ; cf. le commentaire de Merz-Benz, 1990, §§ 5a et 7.

(92) Troeltsch, 1911, p. 101 sq. ; c’est moi qui souligne ; Peter-Ulrich Merz-BenZ.

(93) Troeltsch, 1906, p. 4 ; Troeltsch, 1911, p. 9.

(94) Troeltsch, 1906, p. 4 ; Troeltsch, 1911, p. 9.

(95) Troeltsch, 1906, pp. 4 sq., en particulier p. 7 ; Troeltsch, 1911, pp. 9 sq., en particulier p. 12.

(96) Troeltsch, 1906, p. 7 ; Troeltsch 1911, p. 12.

(97) Troeltsch, 1906, p. 7 ; Troeltsch 1911, p. 12.

(98) Troeltsch, 1906, p. 7 ; Troeltsch 1911, p. 12. 
réfère ici au processus d'auto-élucidation des Lumières inauguré au premier chef par Johann Gottfried Herder. Engagée dans ce processus, la rationalité en vient à engager la réflexion sur sa propre émergence dans la réalité et à buter par ce biais sur "la diversité", i.e. sur la contingence culturelle historique toujours singulière des "jeux de concepts rationnels", "soi-disant" purs et auto-référentiels (99). Ce sont l'autonomie et le caractère relatif des constructions culturelles qui définissent finalement l'individualisme caractéristique du monde moderne. Mais, dans ce cadre, quelle est l'importance qui revient en propre au protestantisme ? Quelle est l'influence absolument originale qu'il a exercée sur ce processus "d'individualisation croissante des convictions, opinions, théories et finalités pratiques" (100) ? À la formation du monde moderne ont pris part les forces historiques concrètes les plus diverses, dont les effets ont été, de surcroît, extrêmement différents dans les domaines de la famille, du droit, de l'État, de l'économie, de la société, de la science et de l'art. Le protestantisme a été impliqué dans la production de chacun de ces effets (101). D'après les "recherches" de Troeltsch, il a "favorisé l'émergence du monde moderne souvent de manière imposante et décisive", sans que, sur aucun des domaines énoncés, il puisse à vrai dire être considéré comme s'il "en" était "purement et simplement le créateur (Schöpfer)" - ainsi que Troeltsch l'ajoute de manière propre à déclencher de nombreuses associations sémantiques (102). La seule chose dont le protestantisme soit le seul responsable, c'est bien plutôt de la "conception de l'âme" fondatrice de l'individualisme moderne (103). L'idée que "l'ensemble de notre monde soit médiatement et immédiatement [imprégné]" par une "métaphysique du personnalisme absolu", vient du protestantisme (104). Avec le protestantisme, "l'idée de liberté, de personnalité, de moi autonome", détient "une assise métaphysique" - qui "exerce son influence même là où elle est combattue et refusée" (105). De l'individualisation, le protestantisme "a directement et délibérément fait un principe" de façonnement de la réalité ; le protestantisme "a dissous le lien qui la [=1'individualisation; $N d T]$ rattachait à une institution séculière hiérarchisée, et l'a rendue disponible pour se fondre librement à tous les intérêts et toutes les forces de la vie" (106). A vrai dire, si l'on veut comprendre en quoi consiste plus précisément cette influence exercée par le protestantisme sur le monde moderne, il convient maintenant d'examiner le phénomène historique qu'est le protestantisme.

(99) Troeltsch, 1906, pp. 7 sq.; Troeltsch, 1911, p. 14.

(100) Troeltsch, 1906, p. 7 ; Troeltsch, 1911, p. 12 ; n'ont pas été ici signalés dans le texte les passages soulignés par Troeltsch; Peter-Ulrich Merz-Benz.

(101) Troeltsch, 1906, pp. 12 sq. ; 29-56; Troeltsch, 1911, pp. 23 ; 46-85.

(102) Troeltsch, 1911, p. 85 ; Troeltsch, 1906, p. 56.

(103) Troeltsch, 1911, p. 21 sq. ; Troeltsch, 1906, pp. $11 s q$.

(104) Troeltsch, 1906, p. 12 ; Troeltsch, 1911, p. 21.

(105) Troeltsch, 1906, p. 12,; Troeltsch 1911, p. 21. [NdT: du texte de Troeltsch ici mobilisé (dans sa version modifiée de 1911), nous suivons pour l'essentiel la traduction française de Marc B. de Launay, cf. Ernst Troeltsch, «Protestantisme et modernité », in Ernst Troeltsch, Protestantisme et modernité, Paris, Gallimard, 1991, pp. 19-129, ici p. 42.]

(106) Troeltsch, 1911, p. 21 ; Troeltsch, 1906, p. 12 ; dans la version de 1906 de la thèse de Troeltsch sur le protestantisme, on ne trouve que la première partie de cette citation. [NdT: DE LAUNAY, op. cit., p. 42.] 
Là encore, c'est entièrement sous l'angle de l'individualisme qu'est déterminé le concept de protestantisme. Néanmoins, une fois ce préalable posé, il apparaît clairement que le concept de protestantisme ne doit en aucune manière être compris comme un phénomène historique global. Le concept de protestantisme se partage d'emblée entre deux formes historiques d'apparition : entre, d'une part, "l'ancien et authentique protestantisme luthérien et calviniste" (107) qui, pour soi, maintient toujours en l'état une culture ecclésiale analogue à celle que connaissait le Moyen Âge ; c'est la puissance d'interprétation de la pure parole biblique qui, bénéficiant de la protection de l'État, a pris la place de l'omnipotente hiérarchie ecclésiale du catholicisme (108) ; et, d'autre part, le néo-protestantisme, composé du baptisme et du spiritualisme, lequel représente effectivement une conviction religieuse "individualiste", voire "subjectiviste" (109). Le néo-protestantisme fait par conséquent lui-même partie du monde moderne. C'est la raison pour laquelle il convient d'attribuer au seul protestantisme ancien la force de changement qui a présidé à la formation du monde moderne (110). Ce qui complique considérablement la question de l'évaluation de l'importance du protestantisme dans l'émergence du monde moderne. Que ce soit à partir d'un point de vue historique général ou du seul point de vue de l'histoire des religions, l'abîme qui sépare le protestantisme ancien du monde moderne apparaît bien trop profond pour qu'un lien direct puisse exister entre eux. D'où ce constat bref et laconique de Troeltsch: "Il n'y a pas de voie directe qui conduirait de la culture ecclésiale du protestantisme à la culture moderne affranchie de toute Église" (111). Et d'ajouter, de manière quelque peu sibylline, que l'importance du protestantisme pour le monde moderne "est à plus d'un titre indirect[e], voire involontaire, et [que] ce qu'il y a malgré tout de commun [à ces deux phénomènes] gît très enfoui dans les profondeurs cachées de la pensée [du protestantisme], [lesquelles profondeurs] ne sont pas immédiatement accessibles à la conscience" (112). Ce qui signifie en clair que l'influence "autonome", "entièrement spécifique et immédiate" exercée par le protestantisme sur l'émergence du monde moderne se situait, 'parmi' différents niveaux de causalité social, politique, économique -, à un niveau purement religieux; pourtant, c'est précisément des profondeurs de sa propre pensée que la religion a été capable de "s'agréger la vie de la culture" et par là même de devenir "une puissance ayant prise sur l'existence" (113). C'est par contre l'idée de la certitude du salut qui est à l'origine de cette influence "structurante" spécifique exercée par le protestantisme sur le monde moderne - à vrai dire, Troeltsch ne se réfère pas au calvinisme mais au luthéranisme.

Accéder à la certitude du salut constitue, pour Luther, quelque chose qui relève strictement de l'individu et qui advient par une "décision simple, radicale et personnelle" (114). À la différence de Calvin, pour Luther cette décision religieuse

(107) Troeltsch, 1906, p. 14 ; Troeltsch, 1911, p. 25. [NdT: De Launay, op. cit., p. 47.]

(108) Troeltsch, 1906, pp. 20 sq. ; Troeltsch, 1911, p. $31 \mathrm{sq.}$

(109) Troeltsch, 1906, p. 15 ; Troeltsch, 1911, pp. 26 sq.

(110) Troeltsch, 1906, p. 17 ; Troeltsch, 1911, p. 30.

(111) Troeltsch, 1906, p. 18; Troeltsch, 1911, p. 31. [NdT: De Launay, op. cit., p. 54].

(112) Troeltsch, 1906, p. 18 ; Troeltsch, 1911, p. 31 sq. [NdT: DE Launay, op. cit., p. 54].

(113) Troeltsch, 1911, p. 87. [NdT: comparer à DE LAUnAY, op. cit., pp. 112-113.]

(114) Troeltsch, 1911, p. 33 ; cf. Troeltsch, 1906, p. 19. [NdT: DE Launay, op. cit., p. 56.] 
ne saurait consister en une option pour un mode de vie rationnel, façonné avec méthode. Refusant toute autorité et toute prescription extérieure au croyant, Luther porte ses regards, de manière exclusive, sur l'élément personnel (115). "Pour la vie personnelle, il avait besoin" - écrit Troeltsch - "d'un élément purement personnel" (116). Par conséquent, si le fait d'accéder à la certitude du salut est un acte personnel, il ne peut, selon Luther, être accompli qu'à l'intérieur de la personne. À quoi vient s'ajouter le fait que, pour Luther, accéder à la certitude du salut équivaut à l'“ascèse", mais dans un sens différent de celui que lui prête Calvin. Pour Luther, l'ascèse est l'attitude qui consiste à s'adapter sans difficulté au cours du monde, et même à s'y résigner. Le croyant vit dans le monde et le surmonte intérieurement parce qu'il ne lui fait nullement confiance, il se "soum[et]" pourtant "humblement à son cours" (117). Même si ce type d'ascèse est pratiqué de façon passive, sans qu'il soit besoin d'être "activiste ni agressif”, à la différence du calvinisme, cela aussi relève de l' "ascèse intramondaine" (118). "Le luthéranisme tolère le monde à travers la croix, la souffrance et le martyre ; le calvinisme maîtrise ce monde pour la gloire de Dieu dans un labeur sans relâche" (119). Le luthérien supporte le monde, en étant au plus profond de luimême (120), ouvert et disposé à accueillir un "miracle", à savoir : la grâce divine qui lui revient. La certitude qu'il a, via cette ascèse intramondaine, d'être prêt à accueillir ce miracle, c'est cela justement pour lui accéder à la certitude du salut en même temps qu'il a confiance en la grâce de Dieu. Avec cette idée de la certitude du salut, quelque chose de décisif s'est passé - ou si l'on veut citer la formulation de Troeltsch : "il en a été de cette construction intellectuelle comme il en va souvent en l'occurrence : la manière nouvelle d'atteindre un but traditionnel devient plus importante que le but même ; à partir de ce qui était biais nouveau se sont développés un but et un contenu eux-mêmes originaux" (121). S'autonomisent alors la "justification subjective et intérieure de la foi" et le fait de supporter personnellement le monde qui va de pair avec cette justification. En cela - selon les mots mêmes de Troeltsch -, "l'évolution du protestantisme" atteignait finalement le "point à partir duquel le moyen, la conviction personnelle, eut plus d'importance que la fin, le salut surnaturel" (122). Ce qui demeurait, c'était le principe de devoir se tourner vers le monde uniquement à partir d'une disposition individuelle et de s'assurer de son existence seulement sur la base de critères autonomes résultant d'un libre choix. En tant que pur principe, l'idée luthérienne de la certitude du salut, cristallisée en une 'forme' spécifique de façonnement de la réalité, pouvait également "se fondre" avec les "intérêts et les forces de la vie" les plus divers (123). Mais l'individualisme se nourrit encore, comme d'un principe, de "l'idée chrétienne" "d'être saisi et formé par l'esprit divin"(124). C'est

(115) Troeltsch, 1906, p. 59 ; Troeltsch, 1911, p. 95.

(116) Troeltsch, 1906, p. 59; Troeltsch, 1911, p. 96. [NdT: De Launay, op. cit., p. 121.]

(117) Troeltsch, 1906, p. 26 ; Troeltsch, 1911, p. 41.

(118) Troeltsch, 1906, pp. 26 sq. ; Troeltsch, 1911, pp. 42 sq. [NdT: DE Launay, op. cit., p. 66.]

(119) Troeltsch, 1906, p. 27 ; cf. Troeltsch, 1911, p. 44. [NdT: De Launay, op. cit., p. 67].

(120) Troeltsch, 1906, p. 59 ; Troeltsch, 1911, p. 96.

(121) Troeltsch, 1906, p. 60 ; Troeltsch, 1911, p. 96. [NdT: DE Launay, op. cit., p. 122.]

(122) Troeltsch, 1906, p. 63 ; Troeltsch, 1911, p. 100. [NdT: De Launay, op. cit., p. 126.]

(123) Troeltsch, 1911, p. 21 ; Troeltsch, 1906, p. 12. [NdT: De Launay, op. cit., p. 42.]

(124) Troeltsch, 1906, p. 12 ; cf. Troeltsch, 1911, p. 21. [NdT: comparer à DE LAUNAY, op. cit., p. 42.] 
précisément cela qui confère à l'individualisme - et avec lui au monde moderne dans sa totalité - une "assise métaphysique" "durable", toujours présente et illimitée (125). Le fait d'avoir 'érigé' l'individualisme en un principe de foi, c'est cela l'influence absolument originale exercée par le protestantisme sur l'émergence du monde moderne. La manière dont Ernst Troeltsch caractérise cette influence constitue le cœur du propos de sa thèse sur le protestantisme.

\section{IV - Protestantisme et monde moderne : un lien de causalité historique et la question de sa saisie conceptuelle}

Est-il possible “d'intégrer" - pour reprendre une question posée plus haut - la thèse de Max Weber sur le protestantisme au complexe causal, examiné par Troeltsch, qui relie le protestantisme au monde moderne ? (126) Que ce qui touche à la thèse de Troeltsch sur le protestantisme soit d'une envergure considérablement plus grande, quant au type d'approche envisagé à tout le moins, que ce qui a trait à la thèse correspondante de Weber, c'est là une chose qui semble évidente. Ce qui occupe Troeltsch, c'est "l'importance effective du protestantisme dans l'émergence de la culture moderne" (127); alors que Weber cherche "seulement à établir si et dans quelle mesure (...) des influences religieuses ont effectivement pris part à l'élaboration qualitative et à l'expansion quantitative [de 'l'esprit' du capitalisme (note de l'A.)] ainsi qu'à constater quels aspects concrets de la culture capitaliste sont référables auxdites influences" (128). Cette affirmation de Weber se trouve à la fin de la première partie de la première version de son étude de 1904 sur le protestantisme. À y regarder de plus près, il ressort cependant que ce qui touche à la thèse wébérienne sur le protestantisme n'est en aucune manière d'une envergure aussi étroite qu'il y paraît dès l'abord et comme on pourrait le croire à la lecture des premières présentations qu'en donne Weber lui-même. Car dans la "note liminaire" qu'il place en tête de ses Gesammelte Aufsätze zur Religionssoziologie de 1920, Weber parle du "capitalisme" en tant qu'il serait "la puissance la plus décisive de notre vie moderne" (129). En accord avec cela, il n'est plus question, à la fin de la première partie de la seconde version de l'étude sur le protestantisme, en date elle aussi de 1920, de "culture capitaliste", mais de "culture reposant sur une base capitaliste" (130). Ce n'est pas tout : à la fin des deux versions de la thèse sur le protestantisme, il est dit respectivement qu'on "peut" et qu'on "pourrait" - après avoir atteint l'objectif de la thèse sur le protestantisme - "tenter d'évaluer dans quelle mesure les contenus culturels modernes sont imputables, suivant leur mode

(125) Troeltsch, 1906, p. 12 ; Troeltsch, 1911, p. 21. [NdT: DE LAUnAy, op. cit., p. 42.]

(126) Troeltsch, 1987 [1910], p. 192.

(127) Troeltsch, 1911, p. 102 ; Troeltsch, 1906, p. 65.

(128) WEBER, 1993 [1904/5], p. 51. Weber.

(129) WeBER, 1988a, p. 4 ; n’ont pas été ici signalés dans le texte les passages soulignés par

(130) WeBER 1988b, p. 83. 
d'émergence historique, à des mobiles religieux, et dans quelle mesure ils sont imputables à d'autres mobiles" (131). Par conséquent, Weber part certes, dans son étude sur le protestantisme, d'une interrogation assez limitée ; il se réserve pourtant la liberté de recourir à cette option qui consisterait à dire qu'avec "l'esprit capitaliste" il a simplement repéré un élément constitutif - voire même l'élément constitutif - de la culture moderne. Prétendre, à partir de là, qu'il s'agit, dans la thèse wébérienne - ainsi que Troeltsch l'affirme et l'expose dans sa propre étude (132) -, de comprendre un "problème relevant" seulement "de l'histoire économique", un "phénomène singulier" à intégrer dans "l'ensemble" des "relations" entretenues par le protestantisme et le monde moderne, c'est affirmer quelque chose d'expressément inexact (133). Troeltsch se trouve ici dans l'erreur, puisqu'à l'évidence il ignore les ambitions explicatives plus vastes énoncées par Weber dès la première version de sa thèse sur le protestantisme. Weber avait dès le départ en tête bien davantage que ce que Troeltsch veut bien lui concéder.

Inversement, on a plutôt l'impression que, dans sa démarche, Troeltsch est plus proche de Weber que ce qu'il est disposé à admettre lui-même. Car la thématique, au départ large, qui a trait à "l'importance effective du protestantisme dans l'émergence de la culture moderne" (134) se trouve sous sa plume très tôt limitée puis, traitée de l'unique point de vue de l'individualisme. De fait, Troeltsch se livre à l'explication de la constitution métaphysique particulière de l'individualisme moderne en tant qu'il représente un principe de façonnement de la réalité - et à rien d'autre. Que ce principe de 'l'individualisme' s'applique aux domaines les plus divers de la vie moderne - la famille, l'État, la société, la science, etc. - pour y prendre à chaque fois une ou des formes spécifiques, cela fait déjà l'objet d'une reconstruction en termes d'histoire universelle; et dans le cadre de cette reconstruction en termes d'histoire universelle, le complexe causal qui relie le protestantisme au monde moderne ne représente, lui aussi, qu'un moment parmi d'autres. Mais il ne peut en aucune manière être question d'affirmer que Troeltsch a procédé à une telle reconstruction en termes d'histoire universelle. La seule et unique chose qui lui importe, c'est bien l'individualisme, voire - pour relier cela à Max Weber - l'individualisme en tant qu'élément constitutif de la culture moderne. Les intentions de Weber et de Troeltsch se rencontrent en fait en un point décisif: de manière analogue à l'esprit capitaliste, l'individualisme est pour Troeltsch cet élément constitutif de la culture moderne qui, via le protestantisme, a été qualitativement élaboré d'une façon particulière, c'est-à-dire métaphysiquement rehaussé et quantitativement étendu, c'est-à-dire intégré aux domaines singuliers les plus différents. Les différences qui se creusent entre Weber et Troeltsch sont néanmoins également évidentes. Car, à la différence de l'esprit capitaliste, l'individualisme n'exerce pas d'emblée d'influence sur un domaine singulier, mais fait son apparition de manière transversale à tous les domaines. Pour Weber, l'influence de l'éthique protestante sur l'esprit capitaliste correspond à l'effet exercé par un élément particulier sur un autre élément particulier, à l'effet exercé par une idée particulière sur une réalité particulière - et c'est seulement au moment où l'esprit

(131) WeBEr, 1993 [1904-1905], p. 51 ; WeBER, 1988b, p. 83 ; c'est moi qui souligne

(132) Troeltsch, 1906, pp. $41 \mathrm{sq}$.; Troeltsch, 1910, p. $66 \mathrm{sq}$.

(133) Troeltsch, 1987 [1910], p. 192.

(134) Troeltsch, 1911, p. 102 ; Troeltsch, 1906, p. 65. 
capitaliste devient la cause de l'émergence des contenus culturels modernes qu'il se généralise ; c'est alors seulement que l'esprit capitaliste acquiert une importance qui touche à l'ensemble de la vie moderne. Pour Troeltsch par contre, l'influence du protestantisme sur l'individualisme correspond d'emblée à l'effet exercé par un élément particulier sur un élément général, à l'effet exercé par une idée particulière sur un principe universel de façonnement de la réalité - et c'est seulement au moment où l'individualisme, métaphysiquement rehaussé, prend une forme concrète dans les différents domaines de la réalité, au moment où il se trouve "amalgamé" aux données réelles les plus diverses, qu'il se particularise. Weber et Troeltsch s'attachent à l'émergence et à la diffusion de la spécificité propre au monde moderne; pour l'un comme pour l'autre, le protestantisme est la cause de l'émergence de cette spécificité. Pourtant, lorsqu'il s'agit de saisir conceptuellement cette spécificité et d'établir la relation qu'elle entretient avec le monde moderne, ils avancent des solutions extrêmement différentes.

Finalement, la thèse d'Ernst Troeltsch sur le protestantisme est entourée d'une légère ironie. Comme on le sait, Troeltsch refuse avec véhémence d'employer des "notions idéales", avant de se contredire et d'y recourir lui-même - à son corps défendant - au fil de son argumentation. En vérité, le concept d"“individualisme" est bien une notion logique idéale qui, du pont de vue de son degré d'abstraction conceptuelle, dépasse de loin les idéauxtypes wébériens. Dans un passage, Troeltsch déclare, de manière explicite et dans le sens conféré à l'idéaltype, que 1" "individualisme" est le "trait essentiel" du monde moderne, sans rendre aucunement compte de la manière dont ce concept a été construit - en particulier sur le rapport qu'il entretient à la réalité. Ce malentendu sur son propre compte a, pour Troeltsch, des conséquences fatales. Car, par là même, l'individualisme ne demeure finalement qu'un principe non spécifié de façonnement de la réalité - un principe à partir duquel aucune voie clairement identifiable ne permet de façonner la facticité, un principe qui ne fournit pas le moindre indice de l'esquisse d'une telle voie. Dans la thèse sur le protestantisme de Troeltsch, une telle voie est pourtant requise; conformément à ce qui y est visé, l'enjeu de l'individualisme est rien moins que 'l'insertion' dans les faits, dans les sphères d'existence de la vie moderne, d'une idée : l'idée luthérienne de la certitude du salut. Cependant, parce qu'il n'explicite pas la manière dont il construit le concept d'individualisme, Troeltsch ne réunit pas les conditions requises par l'élaboration conceptuelle et théorique de ladite 'insertion'. Que l'individualisme doive avoir, dans les faits, un effet structurant, c'est là un principe qu'il s'est contenté d'avancer, mais qu'il n'a pas su démontrer à partir des catégories de pensée mobilisées. Il s'agit pour nous de poursuivre la réflexion de Troeltsch sur le protestantisme - et cela en direction de la sociologie.

Peter-Ulrich MERZ-BENZ

Université de Zurich - Suisse 


\section{BIBLIOGRAPHIE}

Ernst Troeltsch Bibliographie, herausgegeben, eingeleitet und kommentiert von Friedrich Wilhelm Graf und Hartmut Ruddies, Tübingen, JCB Mohr (Paul Siebeck), 1982.

GOTHEIN, Eberhard, Wirtschaftsgeschichte des Schwarzwaldes und der angrenzenden Landschaften. 1. Band: Städte- und Gewerbegeschichte. Herausgegeben von der Badischen Historischen Kommission, Strasbourg, KJ Trübner, 1892.

LEHMANN Hartmut, « Asketischer Protestantismus und ökonomischer Rationalismus. Die Weber-These nach zwei Generationen », in Hartmut LEHMANN, Max Webers “Protestantische Ethik”. Beiträge aus der Sicht eines Historikers, Göttingen, Vandenhoeck und Ruprecht, 1996, pp. 9-29.

MERZ[-BENZ] Peter-Ulrich, Max Weber und Heinrich Rickert. Die erkenntniskritischen Grundlagen der verstehenden Soziologie, Würzburg, éditions Königshausen \& Neumann, 1990.

RACHFAHL Felix, «Kalvinismus und Kapitalismus » (1909), in Max WEBER, Die protestantische Ethik II. Kritiken und Antikritiken, Gütersloh, Mohn 1987, pp. 57-148.

RICKERT Heinrich, Die Grenzen der naturwissenschaftlichen Begriffsbildung. Eine logische Einleitung in die historischen Wissenschaften, Tübingen et Leipzig, JCB Mohr (Paul Siebeck), 1902.

TROELTSCH Ernst, «Was heisst 'Wesen des Christentums'? (1903), in Ernst TROELTSCH, Gesammelte Schriften, vol. 2 (Zur religiösen Lage, Religionsphilosophie und Ethik), Tübingen, JCB Mohr (Paul Siebeck), 1913, pp. 386-451.

TROELTSCH Ernst, «Die Bedeutung des Protestantismus für die Entstehung der modernen Welt», Vortrag, gehalten auf der IX. Versammlung deutscher Historiker zu Stuttgart am 21. April 1906, Historische Zeitschrift, Der ganzen Reihe 97. Band, Dritte Folge - 1. Band, Munich-Berlin, R. Oldenburg, 1906, pp. 1-66.

TROELTSCH Ernst, Die Bedeutung des Protestantismus für die Entstehung der modernen Welt, MunichBerlin, R. Oldenburg, 1911.

TROELTSCH Ernst, «Die Kulturbedeutung des Calvinismus », in Max WEBER, Die protestantische Ethik II. Kritiken und Antikritiken, Gütersloh, Mohn 1987, pp. 188-215.

TROELTSCH Ernst, "Protestantisme et modernité», in Ernst TROELTSCH, Protestantisme et modernité, Paris, Gallimard, 1991.

WEBER Max, « Die protestantische Ethik und der 'Geist' des Kapitalismus. I. Das Problem », Archiv für Sozialwissenschaft und Sozialpolitik, hrsg. v. W. Sombart, M. Weber und E. Jaffé, 20. Band, 1. Heft, Tübingen, JCB Mohr (Paul Siebeck), 1904, pp. 1-54. [Das 1. Heft des 20. Bandes erschien im November des angegebenen Jahres.]

WEBER Max, «Die protestantische Ethik und der 'Geist' des Kapitalismus. II. Die Berufsidee des asketischen Protestantismus », Archiv für Sozialwissenschaft und Sozialpolitik, hrsg. v. W. Sombart, M. Weber und E. Jaffé, 21. Band, 1. Heft, Tübingen, JCB Mohr (Paul Siebeck), 1905, pp. 1-110. [Das 1. Heft des 21. Bandes erschien ca. im Juni des angegebenen Jahres.]

WEBER Max, "Antikritisches zum 'Geist' des Kapitalismus », in Max WEBER, Die protestantische Ethik II. Kritiken und Antikritiken, Gütersloh, Mohn, 1987, pp. 149-187.

WEBER Max, Gesammelte Ausfsätze zur Wissenschaftslehere, Vierte, erneut durchgesehene Auflage, herausgegeben von Johannes Winckelmann, Tübingen, JCB Mohr (Paul Siebeck), 1973.

WEBER Max, «Vorbemerkung », in Max WEBER, Gesammelte Ausfsätze zur Religionssoziologie I, Tübingen, JCB Mohr (Paul Siebeck), 1988(a), pp. 1-16.

WEBER Max, "Die protestantische Ethik und der 'Geist' des Kapitalismus », in Max WEBER, Gesammelte Ausfsätze zur Religionssoziologie I, Tübingen, JCB Mohr (Paul Siebeck), 1988(b), pp. 17-206.

WEBER Max, Die protestantische Ethik und der 'Geist' des Kapitalismus. Textausgabe auf der Grundlage der ersten Fassung von 1904/1905 mit einem Verzeichnis der wichtigsten Zusätze und Veränderungen aus der zweiten Fassung von 1920, herausgegeben und eingeleitet von Klaus Lichtblau und Johannes Weiß, Weinheim, Beltz Athenäum, 1993. 


\section{Résumé}

Un des modèles explicatifs les plus connus et les plus importants de l'émergence du monde moderne et de l'essor du "capitalisme » est la thèse de Max Weber sur le protestantisme. Moins connue en revanche est la thèse sur le protestantisme qu'Ernst Troeltsch, un collègue et ami de Weber à Heidelberg, a également développé quelques années seulement après ce dernier. Selon Troeltsch, le protestantisme serait responsable de la montée de l'individualisme, un des éléments constitutifs majeurs de la culture moderne. Pour autant, alors que Troeltsch considère le protestantisme, dès ses débuts, comme une partie intégrante de l'histoire universelle - c'est la raison pour laquelle il lui attribue une importance universelle-, Weber, lui, voit dans le protestantisme et dans l'influence qu'il a exercée sur la conduite des hommes, un phénomène singulier qui doit la place qu'il s'est acquise dans le monde moderne à l'émergence du capitalisme. Compte tenu de ces différences d'analyses, particulièrement intéressant et riche d'enseignements est le fait que Weber et Troeltsch s'appuient tous deux sur la "logique de l'histoire " élaborée par Heinrich Rickert. Les thèses sur le protestantisme de Weber et de Troeltsch peuvent ainsi être comprises comme des variations sur la philosophie de Rickert qui s'efforceraient l'une comme l'autre de saisir le lien de causalité entre le protestantisme et le monde moderne.

\section{Abstract}

One of the most popular and altogether most important explanations for the genesis of the modern world with its capitalist order is Max Weber's study on protestantism. But there is another, almost unknown explanation that Weber's friend and colleague, Ernst Troeltsch, published only a few years after Weber's. According to Troeltsch, protestantism is responsible for the development of individualism as a significant feature of modern culture. But in Troeltsch's view protestantism has been part of global history from its very beginning. That is why he attributed universal significance to this religion. Weber, in contrast, understood protestantism, and its influence on the human conduct of life, as a single phenomenon which gains its importance for the modern world from capitalism. Given this contrast, it is especially interesting to see that both Weber and Troeltsch used Heinrich Rickert's philosophy of history to develop their positions. Their theories on protestantism, therefore, can be reconstructed as variations of Rickert's philosophy, trying to grasp the causal connection between protestantism and the modern world.

\section{Resumen}

Uno de los modelos explicativos más conocidos, y más importantes, de la emergencia del mundo moderno y del surgimiento del "capitalismo" es la tesis de Max Weber sobre el protestantismo. Menos conocida en cambio es la tesis sobre el protestantismo que Ernst Troeltsch, colega y amigo de Weber en Heidelberg, desarrolla sólo algunos años más tarde. Según Troeltsch, el protestantismo sería responsable del aumento del individualismo como elemento constitutivo mayor de la cultura moderna. Pero mientras que Troeltsch considera al protestantismo, desde el principio, como una parte integrante de la historia universal - y es ésta la razón por la cual le atribuye una importancia universal -, Weber, en cambio, ve en el protestantismo y en la influencia que ha ejercido sobre la conducta de los hombres, un fenómeno singular que debe su importancia en el mundo moderno a la emergencia del capitalismo. Consideradas estas diferencias de análisis, es en sí particularmente interesante y rico en enseñanzas el hecho que tanto Weber como Troeltsch se apoyan en la "lógica de la historia" elaborada por Heinrich Rickert. Las tesis sobre el protestantismo de Weber y de Troeltsch pueden así ser entendidas como variantes de la filosofía de Rickert que se esforzarian tanto una como la otra de entender el lazo de causalidad entre el protestantismo y el mundo moderno. 
